\title{
Entrenamiento Neuromotor en pacientes ancianos pluripatológicos en las Unidades de Hospitalización a Domicilio: estudio piloto
}

\section{Neuromotor Training in elderly multi-pathological patients in the Home Hospitalization Units: a pilot study}

\section{Treinamento neuromotor em pacientes idosos multipatológicos nas Unidades de Internação Domiciliar: estudo piloto}

\author{
Blasco-Lafarga, C. ${ }^{1}$, Sanchis-Sanchis, R. ${ }^{1}$, Sanchis-Soler, G. ${ }^{2}$, San Inocencio-Cuenca, D. ${ }^{3}$, Llorens, P. ${ }^{3}$ \\ ${ }^{1}$ Departamento de Educación Física y Deportiva, Universidad de Valencia (España); \\ ${ }^{2}$ Departamento de Didáctica General y Didácticas Específicas, Universidad de Alicante (España); \\ ${ }^{3}$ Servicio de Urgencias-UCE-UHD, Hospital General de Alicante (España).
}

\begin{abstract}
RESUMEN
Introducción: El envejecimiento se acompaña de factores de riesgo cuya reducción mejora la supervivencia. La actividad física incide sobre ellos, lo que realza su papel preventivo y su valor como tratamiento no-farmacológico. Dada la escasez de programas de entrenamiento específicos para ancianos pluripatológicos, sobre todo durante el periodo de inicio de resolución de la actividad clínica de una enfermedad aguda, o de la exacerbación de una o varias de las enfermedades crónicas, el presente estudio piloto analiza la viabilidad y efectos de un programa corto de entrenamiento neuromotor en estos pacientes ingresados en UHD (Unidad de Hospitalización Domiciliaria). Método: 11 ancianos pluripatológicos (82,2 $\pm 6,9$ años, 8 hombres) completaron 4 semanas de entrenamiento funcional con orientación neuromuscular y cognitiva, con evaluación pre-post sobre capacidad funcional (equilibrio, marcha, fuerza de tren inferior y agilidad), composición corporal y calidad de vida -6 semanas, incluida la evaluación-. Se realizaron dos sesiones domiciliarias supervisadas y una tercera autónoma semanalmente. Resultados: Los ancianos mejoraron en equilibrio y marcha (Tinetti-total: $14,0 \pm 9,0$ vs $17,9 \pm 7,3$ puntos; $p=0,007$ ), fuerza (test sentarse y levantarse 30 -s: $3,0 \pm 3,7$ vs 4,1 $\pm 4,1$ reps.; $p=0,034$ ), agilidad (TUG-test categorizado; $p=0,001$ ) y peso $(82,2 \pm 14,0 \mathrm{vs} 79,2 \pm 13,9 \mathrm{~kg}$; $\mathrm{p}=0,036$ ), aunque no en calidad de vida relacionada con la salud (SF-36-v2: $32,9 \pm 7,8$ vs $31,2 \pm 7,7$ puntos; $p=0,722$ ). La presencia del técnico aumentó el cumplimiento un 20\%. Conclusiones: El dinamismo de UHD es idóneo para implantar programas de actividad física especializada que frenen los efectos devastadores del binomio envejecimiento-inactividad. Una corta duración no les resta eficacia.

Palabras clave: Aptitud física, calidad de vida, envejecimiento, fragilidad, paciente geriátrico, prevención primaria.
\end{abstract}

\section{ABSTRACT}




\section{Blasco-Lafarga, C., Sanchis-Sanchis, R., Sanchis-Soler, G., San Inocencio-Cuenca, D., Llorens, P.}

Introduction: Aging is linked to a various risk factors whose reduction improves survival. Physical activity affects many of them, what enhances its preventive role and its value as a non-pharmacological treatment. Given the scarcity of specific training programs for elderly people with multiple pathologies, especially during the start period of resolution of the clinical activity of an acute disease, or the exacerbation of one or several of the chronic diseases, the present pilot study aims to analyze the feasibility and effects of a short neuromotor training program in these patients admitted to UHD (Home Hospitalization Unit). Methods: 11 elderly patients (82.2 \pm 6.9 years, 8 men; acute phase of disease) completed 4 weeks of a neuromuscular and cognitive orientated functional training program, with pre-post assessment of functional capacity (i.e. balance, gait speed, lower limb strength and agility), body composition and quality of life -6 weeks, including the evaluation-. There were 2 supervised sessions and a third autonomous session every week. Results: Significant improvements were found in balance and gait (Tinetti-total: $14.0 \pm 9.0$ vs $17.9 \pm 7.3$ points; $p=0.007)$, strength ( 30 -seconds Chair Stand Test: $3.0 \pm 3 ; p=0.001)$, agility (TUG-test categorized; $\mathrm{p}=0.001)$ and weight $(82.2 \pm 14.0 \mathrm{vs} .79 .2 \pm 13.9 \mathrm{~kg} ; \mathrm{p}=0.036)$, but not in health-related quality of life (SF-36-v2: $32.9 \pm 7.8$ vs $31.2 \pm 7.7$ points; $\mathrm{p}=0.722$ ). The technician's presence increased adherence to the program by $20 \%$. Conclusions: The dynamism of UHD is ideal for implementing specialized physical activity programs that counteract the devastating effects of the binomial aging-inactivity. A short duration does not reduce effectiveness. Keywords: Aging, frailty, geriatric patient, physical fitness, primary prevention, quality of life.

\section{RESUMO}

Introdução: $O$ envelhecimento é acompanhado por fatores de risco cuja redução melhora a sobrevida. A atividade física os afeta, o que aumenta seu papel preventivo e seu valor como tratamento não farmacológico. Dada a escassez de programas específicos de treinamento para pacientes idosos, especialmente durante o período da iniciação da resolução da atividade clínica de uma doença aguda, ou da exacerbação de uma ou mais das doenças crônicas, o presente estudo piloto analisa a viabilidade e os efeitos de um programa curto de treinamento neuromotor nesses pacientes internados em UHD (Unidade de Internação Domiciliar). Método: 11 idosos com múltiplas patologias (82,2 $\pm 6,9$ anos, 8 homens, doença de fase aguda) completaram 4 semanas de treinamento funcional com orientação neuromuscular e cognitiva, com avaliação pré-pós de capacidade funcional (equilíbrio, marcha, menor força e agilidade do trem), composição corporal e qualidade de vida -6 semanas, incluindo avaliação-. Foram realizadas duas sessões domiciliares supervisionadas e uma sessão semanal autônoma. Resultados: Os idosos melhoraram em equilíbrio e marcha (Tinetti-total: $14,0 \pm 9,0$ vs $17,9 \pm 7,3$ pontos, $\mathrm{p}=0,007$ ), força (teste de sentar e levantar 30 -s: $3,0 \pm 3,7$ vs $4,1 \pm 4,1$ repetições, $\mathrm{p}=0,034)$, agilidade (teste categorizado pelo TUG, $\mathrm{p}=0,001)$ e peso $(82,2 \pm 14,0$ vs

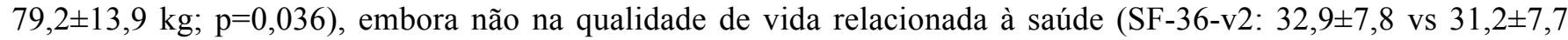
pontos, $\mathrm{p}=0,722$ ). A presença do técnico aumentou a conformidade em $20 \%$. Conclusões: O dinamismo da UHD é ideal para a implementação de programas especializados de atividade física que neutralizam os efeitos devastadores do binômio envelhecimento-inatividade. Uma curta duração não reduz a eficácia.

Palavras chave: Aptidão física, envelhecimento, fragilidade, paciente geriátrico, prevenção primária, qualidade de vida.

\section{INTRODUCCIÓN}




\section{Entrenamiento Neuromotor en pacientes ancianos pluripatológicos}

Durante la vejez, cualquier agresión aparentemente pequeña sumada a una situación de fragilidad se traduce en un cambio desproporcionado en la salud del anciano (Clegg, Young, Iliffe, Rikkert, y Rockwood, 2013). Este hecho se acompaña de un empeoramiento sintomático en los ancianos con patología crónica, con el consiguiente aumento en el riesgo de hospitalización. Las Unidades de Corta Estancia (UCE) se convierten, entonces, en una alternativa potente frente a la Hospitalización Convencional (Epelde, Iglesias-Lepine, y Anarte, 2012; Guirao Martínez, Sempere Selva, López Aguilera, Sendra Pina, y Sánchez Payá, 2008). Los ingresos en UCE permiten tratamientos agudos sin merma de la eficiencia, contienen la tasa de infecciones nosocomiales, y mejoran la atención percibida por el paciente, sin repercusión sobre el aumento de los reingresos y la mortalidad (Epelde et al., 2012), aunque su eficacia disminuye con la edad (Guirao Martínez et al., 2008). Además, se sabe que pueden reportar de forma secundaria problemas cognitivos, emocionales o sociales en los ancianos pluripatológicos o paliativos ingresados (Sisamón, 2012). Por ello las Unidades de Hospitalización Domiciliaria (UHD) pretenden ir un paso más allá en la atención centrada en la persona, y aspiran a ser una vía eficaz en la contención de este problema (Blasco-Lafarga, Sanchis-Soler, Sanchis-Sanchis, y Valencia-Peris, 2015; Mas y Santaeugènia, 2015).

La calidad de los servicios en UHD y su correcta integración resulta clave en la reducción/interrupción del riesgo de deterioro funcional en pacientes frágiles. $\mathrm{Su}$ visión de alta precoz, basada en intervenciones complejas y un seguimiento individualizado, integral y pluridisciplinar, parece especialmente interesante en el caso de los ancianos pluripatológicos o paliativos (Mas y Santaeugènia, 2015). Igualmente beneficioso sería aumentar su potencial preventivo y de identificación precoz de los síntomas de la fragilidad en esta población. Sin embargo, todavía no son frecuentes las intervenciones en UHD relacionadas con la prescripción de ejercicio físico o el fomento de la práctica de actividad física en estos momentos de fase aguda de la enfermedad, a pesar de su menor coste frente a muchos tratamientos farmacológicos (Garrett et al., 2011), y de haberse constatado, además, su valor como uno de los pilares básicos en la contención de la fragilidad (Casas-Herrero, Cadore, Martínez-Velilla, y Izquierdo, 2015; Casas Herrero y Izquierdo, 2012;
Clegg y Young, 2011; Garrett et al., 2011; Izquierdo et al., 2016).

En los últimos años se han desarrollado numerosos avances en intervenciones nutricionales y farmacológicas en relación a la fragilidad, algunas de ellas con ejercicio físico, siendo la presencia de este último la que ha reportado mejores resultados en la prevención del deterioro funcional (Abizanda Soler, 2010; Izquierdo et al., 2016). Además, hoy se sabe que algunos de los factores de riesgo más importantes para la longevidad de la población adulta son altamente modificables (Yates, Djoussé, Kurth, Buring, y Gaziano, 2008). La hipertensión, la diabetes, la obesidad, el tabaquismo o el sedentarismo pueden condicionar con su presencia o ausencia que la probabilidad de vivir hasta los 90 años se reduzca del 54 al 4\% una vez cumplidos los 79 (Yates et al., 2008). No obstante, sus consecuencias negativas son altamente sensibles frente a los cambios de hábitos, los estilos de vida activos, los programas de ejercicio físico o los cuidados nutricionales, con más beneficios sobre la contención de los riesgos cuanto más temprana es su implantación (Yates et al., 2008). Ello refuerza la importancia de la educación y la prevención. Asumir que la reducción de estos factores de riesgo puede prolongar la supervivencia y mejorar los años de vida vividos con calidad otorga todo su sentido a la atención sanitaria con carácter preventivo. $\mathrm{Y}$ es en este contexto donde la función preventiva de la actividad física, junto a su papel como "tratamiento no farmacológico" (Izquierdo et al., 2016; Vogel et al., 2009), cobra especial relevancia para la población que envejece, con especial atención a sus beneficios sobre la reducción de los procesos inflamatorios, en la base de muchas enfermedades asociadas al adulto mayor (Woods, Vieira, y Keylock, 2009).

Es, por tanto, pertinente preguntarse por la posibilidad y resultado de implantar programas de ejercicio específicos para estos ancianos pluripatológicos, una vez probado el potente efecto de este último en la contención de la enfermedad, la mejora de la calidad de vida y la capacidad funcional en esos pacientes (Pedersen y Saltin, 2015). Muchos servicios incorporan el ejercicio como herramienta rehabilitadora (Matsuda, Shumway-Cook, y Ciol, 2010), pero escasean las intervenciones basadas en programas de entrenamiento holístico, buscando respuestas integrales y una activación física global, 
Blasco-Lafarga, C., Sanchis-Sanchis, R., Sanchis-Soler, G., San Inocencio-Cuenca, D., Llorens, P.

neuromotora, afectiva y específica, aunque se sabe que los programas multicomponente que combinan ejercicio físico (aeróbico, fuerza y equilibrio) y tareas cognitivas potencian sus efectos de forma sinérgica, $y$ aumentan sus beneficios (Di Benedetto, Müller, Wenger, Düzel, y Pawelec, 2017).

Así pues, el objetivo del presente estudio piloto es analizar la viabilidad y efectos de un programa corto de entrenamiento neuromotor, domiciliario, supervisado e individualizado, sobre un grupo de ancianos pluripatológicos ingresados en UHD. El estudio, coordinado por un equipo multidisciplinar de profesionales, ha sido realizado durante la etapa de inicio de resolución clínica o de actividad de la enfermedad o enfermedades que motivaron su ingreso, pero todavía con signos y síntomas activos propios de la etapa clínica de proceso.

\section{MATERIAL Y MÉTODOS}

\section{Pacientes}

Atendiendo a las recomendaciones de OlleroBaturone, Sanz-Amores, y Padilla-Marín (2016), se seleccionaron pacientes ancianos con patología crónica ingresados en la UHD del Hospital General de Alicante, derivados por el personal médico. Como criterios de inclusión: (I) paciente $\geq 65$ años (II) con patología crónica; (III) apto física (capaz de mantenerse en sedestación) y mentalmente (sin deterioro cognitivo o con deterioro leve, esto es, MEC$30 \geq 20$ ) para la actividad física (AF) (derivación médica); (IV) con consentimiento informado. Como criterios de exclusión: (I) paciente con patología incompatible con la AF (decisión médica); (II) que ya sigue un programa de ejercicio físico (EF) regular antes del ingreso; (III) recibe rehabilitación fisioterapéutica o terapia ocupacional; (IV) y muestra asistencia discontinua (es decir, la no realización de ejercicio físico durante más de dos sesiones consecutivas sin causa médica justificada). El estudio respeta las normas éticas concordantes con la declaración de Helsinki, y fue aprobado por el Comité de Ética del Hospital General de Alicante y de la Universidad de Valencia. Todos los pacientes fueron informados sobre la intervención y firmaron su consentimiento previamente.
Estudio cuasi-experimental de corte longitudinal, con un solo grupo sujeto a tratamiento y dos evaluaciones (pre y post programa), con el fin de analizar los cambios en fuerza de tren inferior (30-seconds Chair Stand Test: $30 s-C S T)$; equilibrio estático, dinámico y calidad de marcha (Tinetti Test); agilidad (Timed UpAnd-Go Test: TUG); composición corporal (bioimpedancia, Tanita ${ }^{\circledR}$ BC545N); y calidad de vida relacionada con la salud (cuestionario $S F-36-v 2$ ). Diseño intrasujeto con el programa de entrenamiento neuromotor combinado con el seguimiento médicofarmacológico habitual como único factor.

\section{Descripción de las pruebas}

El TUG (Rikli y Jones, 1999) requiere que el paciente se levante desde la posición de sentado, camine 2,44 metros lo más rápido y seguro que pueda hasta un pequeño señalizador situado en el suelo frente a su silla, dé media vuelta y regrese para sentarse de nuevo en ella en el menor tiempo posible. De acuerdo con sus autoras, se realizó la prueba 2 veces, con un intento previo de familiarización, contando para el análisis el mejor tiempo entre ambas. El test se grabó en vídeo y, posteriormente, se analizó mediante el software Kinovea ${ }^{\circledR}$. El inicio del registro del tiempo se definió como el primer movimiento del paciente para levantarse tras oír la señal sonora. Y el final del tiempo como el momento en el que los glúteos se apoyaban por completo sobre el asiento.

En cuanto al 30s-CST (Rikli y Jones, 1999), el test consiste en contar el número de veces que un individuo puede levantarse, desde la posición de sentado y sin ayudarse de los brazos, en 30 segundos. El test se realizó una sola vez, con 2 repeticiones previas de prueba para asegurarse que el paciente había entendido las instrucciones y que realizaba el movimiento correctamente. Se contaron movimientos completos (levantarse y sentarse por completo en la silla), desde la primera señal sonora, que marcaba el inicio de la cuenta atrás, hasta la segunda señal sonora, que indicaba el final de la prueba.

Ambas pruebas motrices fueron precedidas $48 \mathrm{~h}$ antes de la evaluación de la marcha y el equilibrio (Tinetti Test (Tinetti, Franklin Williams, y Mayewski, 1986)), como primeros indicadores de la funcionalidad de los pacientes. El test utiliza movimientos que son habituales en situaciones de la vida cotidiana, y la versión utilizada en este estudio incluye un total de 16 


\section{Entrenamiento Neuromotor en pacientes ancianos pluripatológicos}

ítems (9 para el equilibrio y 7 para la marcha), pudiendo obtener una puntuación máxima de 28 puntos.

Como se ha señalado, la evaluación incluyó además el testeo de la composición corporal mediante bioimpedancia: Tanita $^{\mathbb{B}}$ BC545N, modelo con sistema tetrapolar (tarso-metatarso y carpo-metacarpo) y una corriente de medición de $50 \mathrm{kHz}$ y $100 \mu \mathrm{A}$, con una precisión de $0,1 \mathrm{~kg}$ para el peso y $0,1 \%$ para la estimación de la grasa corporal. Y la evaluación de la calidad de vida relacionada con la salud mediante el cuestionario SF-36 (Alonso, Prieto, y Antó, 1995), compuesto por 36 preguntas que valoran los estados de la salud, tanto positivos como negativos. Este fue administrado por el mismo evaluador en todos los casos. Las versiones del cuestionario varían en cuanto al periodo recordatorio: la estándar (4 semanas; utilizada en este estudio) y la aguda (1 semana).

\section{Intervención y protocolo: el programa $E F A M-U V^{\odot}$}

Durante 4 semanas se realizaron 2 sesiones de entrenamiento dirigidas y 1 autónoma, con una separación mínima de 48 horas entre ellas. En total, 3 sesiones personalizadas de 45 minutos, diseñadas y supervisadas por profesionales especialistas en Educación Física (EF) para adultos mayores (CAFD). El programa se ejecutó según las directrices del Método de entrenamiento funcional cognitivo neuromotor EFAM-UV $V^{\odot}$ (Blasco-Lafarga et al., 2016), tal y como se adapta al ámbito hospitalario (SanchisSoler, 2017; Sisamón, 2012). Según este método, cada sesión se compuso de: activación (fuerza con movilidad articular y educación respiratoria), bloque de trabajo neuromuscular (fuerza orientada a la mejora de la musculatura implicada en la marcha extremidades inferiores- y el control postural -core-), y entrenamiento de habilidades motrices (realizadas desde la reeducación del paso), todo ello bajo la metodología de la doble tarea para incidir sobre las habilidades cognitivas -función ejecutiva-. De acuerdo con los patrones motrices más comunes en los programas de tercera edad, resumidos por Saüch y Castañer (2014), en este contexto el programa EFAM$\mathrm{UV}^{\mathcal{O}}$ adoptó una forma de programa psicomotor, de perfil utilitario y terapéutico, en el que se combinaron gran número de implementos para potenciar la estimulación, y se incidió de forma conjunta sobre los tres grupos de habilidades motrices básicas (estabilidad, locomoción y manipulación). A nivel de capacidades perceptivo-motrices se trabajó básicamente sobre la coordinación segmentaria y la coordinación en equilibrio dinámico, así como sobre la fuerza y la movilidad a nivel de capacidades físicomotrices (Saüch y Castañer, 2014). La orientación espacio-temporal también se incluía entre las consignas de las tareas. Se utilizó para ello picas, mancuernas, elásticos, pelotas de espuma, cuerdas, conos chinos y otros marcadores, así como steps, superficies inestables y en general material pequeño de entrenamiento neuromuscular susceptible de ser utilizado en un domicilio. La velocidad de la contracción fue variable, tratando de utilizar todo el espectro de amplitud de rangos de velocidad dentro de las posibilidades del paciente. El número de repeticiones osciló entre 2 y 8 según objetivos y velocidades, con intensidades del Esfuerzo Percibido (EP) en torno a 4-5 y la escala analógica del dolor (Escala EVA) de 2-3, de nuevo según objetivos e individuos. Para optimizar el registro de EP se utilizó la escala con pictogramas de Da Silva-Grigoletto et al. (2013). Como apoyo al control de la intensidad se controlaron la frecuencia cardiaca (FC), la tensión arterial (TA) y la saturación de oxígeno $(\mathrm{SaO} 2)$ (Sanchis-Soler, 2017; Sisamón, 2012).

Todas las sesiones fueron diseñadas de acuerdo a principios básicos de entrenamiento (Casas Herrero y Izquierdo, 2012) como el principio de la sobrecarga, de la progresión, de la especificidad y la individualidad del entrenamiento, y del desentrenamiento o reversibilidad. En cuanto a las evaluaciones pre y post programa, se llevaron a cabo en el domicilio del paciente las semanas anterior y posterior al entrenamiento, con evaluadores previamente entrenados para optimizar las mediciones. Las pruebas fueron divididas en 2 días diferentes, con una separación de $48 \mathrm{~h}$ entre ellas, y siempre en horarios y condiciones similares para los mismos sujetos. El primer día de evaluación se realizaron Tanita ${ }^{\circledR}, S F-36$ y Tinetti Test, y el segundo día $T U G$ y $30 s-C S T$, en el orden citado.

\section{Análisis estadístico}

Al ser un estudio piloto no se realizó una estimación muestral previa. Tras comprobar la distribución (Shapiro-Wilk), y debido al pequeño tamaño de la muestra, se utilizó la prueba de Wilcoxon para comparar medias en muestras relacionadas. Además, se categorizó el $T U G$ como alternativa a la evaluación 


\section{Blasco-Lafarga, C., Sanchis-Sanchis, R., Sanchis-Soler, G., San Inocencio-Cuenca, D., Llorens, P.}

del tiempo de ejecución en el test (Blasco-Lafarga, Sanchis-Sanchis, Sanchis-Soler, y Llorens-Soriano, 2014), dado el bajo nivel de condición física de los pacientes y los cambios observados en las ayudas necesarias para su realización tras el programa. Las diferencias entre categorías pre-post intervención (de menos a más: no puede hacer el test, usa andador, usa bastón, no necesita ayuda), se analizaron mediante jiCuadrado. Se utilizó el paquete estadístico IMB SPSS Statistics para Windows, versión 22.0 (Armonk, NY: IBM Corp), con un nivel de significación estadística de $p<0,05$ (intervalo de confianza del 95\%).

\section{RESULTADOS}

La Tabla 1 muestra las características sociodemográficas y clínicas de los 11 pacientes $(82,2$ $\pm 6,9$ años, 8 hombres y 3 mujeres) que completaron el estudio. Como muestra la Figura 1, el programa de entrenamiento $E F A M-U V^{\circ}$ produjo mejoras significativas $(p<0,05)$ en fuerza de tren inferior, equilibrio estático y dinámico, marcha y peso. Los cambios no fueron significativos en IMC $(30,06 \pm 3,76$ vs $29,80 \pm 4,73)$, porcentaje de grasa corporal $(37,29$ $\pm 9,72$ vs $36,22 \pm 8,68)$, masa muscular $(47,83 \pm 11,24$ vs $48,12 \pm 8,35 \mathrm{~kg}$.), y masa ósea $(2,54 \pm 0,55$ vs 2,58 $\pm 0,41)$.

El TUG categorizado reveló mejoras significativas en agilidad $\left(\chi^{2}(6)=22,00 ; p=0,001\right)$ pese a no registrar reducciones respecto al tiempo empleado en la ejecución $(29,18 \pm 17,93$ vs $33,25 \pm 22,85 \mathrm{seg}$.). De las 4 categorías mencionadas, 3 pacientes pasaron de "No puede hacer el test" a la categoría "usa andador" $(27,3 \%) ; 1$ pasó de "usa andador" a "usa bastón" $(9,1 \%) ;$ y 1 de "usa bastón" a "no necesita ayuda" $(9,1 \%)$. El 54\% restante no cambió de grupo ("no necesita ayuda"). Ningún sujeto experimentó un deterioro en sus capacidades para realizar el $T U G$.

Finalmente, los cambios en el $S F-36$ no fueron significativos (Figura 1). El hecho de que el programa se desarrollara en el propio domicilio del paciente y de forma supervisada permitió una asistencia $\mathrm{y}$ cumplimiento superior al $90 \%$ en las sesiones dirigidas, que sin embargo cayó por debajo del $70 \%$ en las sesiones autónomas.
Tabla 1. Características sociodemográficas y clínicas basales de los pacientes.

\begin{tabular}{lc} 
& $\begin{array}{c}\text { Total de } \\
\text { pacientes } \\
(\mathrm{n}=11)\end{array}$ \\
\hline Edad, años (media \pm desviación típica) & $82,2 \pm 6,9$ \\
Peso $^{\mathrm{a}}, \mathrm{kg}($ media \pm desviación típica) & $82,2 \pm 14$ \\
$\mathrm{IMC}^{\mathrm{a}}$ (media \pm desviación típica) & $30,1 \pm 3,8$ \\
$\left.\mathrm{Sexo}^{\mathrm{n}}(\%)\right]$ & \\
Masculino & $8(72,7 \%)$ \\
Femenino & $3(27,3 \%)$ \\
Diagnóstico de ingreso [n (\%)] & \\
Cardiovascular & $5(45,5 \%)$ \\
Endocrino y/o Metabólico & $5(45,5 \%)$ \\
Respiratorio & $4(36,4 \%)$ \\
Digestivo & $1(9,1 \%)$ \\
Otro & $2(18,2 \%)$ \\
Deterioro cognitivo ${ }^{\mathrm{b}}$ [n (\%)] & \\
Con deterioro & $3(27,3 \%)$ \\
Sin deterioro & $8(72,7 \%)$ \\
Nivel de dependencia ${ }^{\mathrm{c}}$ [n (\%)] & \\
Dependencia total & $1(9,1 \%)$ \\
Dependencia severa & $5(45,5 \%)$ \\
Dependencia moderada & $2(18,2 \%)$ \\
Dependencia escasa & $2(18,2 \%)$ \\
Independencia & $1(9,1 \%)$ \\
\hline
\end{tabular}

\footnotetext{
${ }^{\text {a }} 2$ pacientes no pudieron pesarse por no poder mantenerse de pie sin ayuda.

${ }^{\mathrm{b}}$ Medido mediante el Mini Examen Cognoscitivo de Lobo, versión de 30 preguntas (Punto de corte: $\leq 23$ puntos).

${ }^{c}$ Medido mediante el Índice de Barthel (0-20: dependencia total; 21-60: severa; 61-90: moderada; 91-99: escasa; 100: independencia).
}

\section{DISCUSIÓN}

A pesar de lo reducido de su duración y tamaño muestral, el presente estudio piloto sugiere la viabilidad y efectos positivos de introducir programas de entrenamiento neuromotor como EFAM-UV (Blasco-Lafarga et al., 2016) en las UHD. Las mejoras obtenidas en fuerza del tren inferior y equilibrio sugieren que la orientación neuromuscular y cognitiva puede ser adecuada y eficaz para entrenar en sus domicilios a este tipo de población, de forma similar a otras intervenciones (Casas Herrero y Izquierdo, 2012; Sanchis-Soler, 2017), pues aunque las mejoras son pequeñas, la intervención fue corta y se partía de niveles basales muy bajos comparados con pacientes sanos (Rikli y Jones, 2013). Conociendo la 


\section{Entrenamiento Neuromotor en pacientes ancianos pluripatológicos}

importancia que tienen estos factores como predictores de caídas y pérdida de funcionalidad (Asmidawati, Hamid, Hussain, y Hill, 2014; Hafström, Malmström, Terdèn, Fransson, y Magnusson, 2016; Roldán-Jiménez, Bennett, y Cuesta-Vargas, 2015), estos hallazgos justificarían la necesidad de realizar en un futuro un estudio con una mayor muestra y un grupo control aleatorizado para comprobar la viabilidad real de integrar programas de entrenamiento temprano en el ámbito hospitalario, aun siendo de corta duración. Se reafirma, por tanto, la propuesta de tratar a un síndrome tan complejo como el de la fragilidad mediante intervenciones multifactoriales (Casas Herrero y Izquierdo, 2012), dando especial importancia al trabajo de fuerza y equilibrio. Esta orientación más neuromuscular habría permitido conseguir en sólo 4 semanas resultados semejantes a intervenciones similares de mayor duración (Liubicich, Magistro, Candela, Rabaglietti, y Ciairano, 2012).
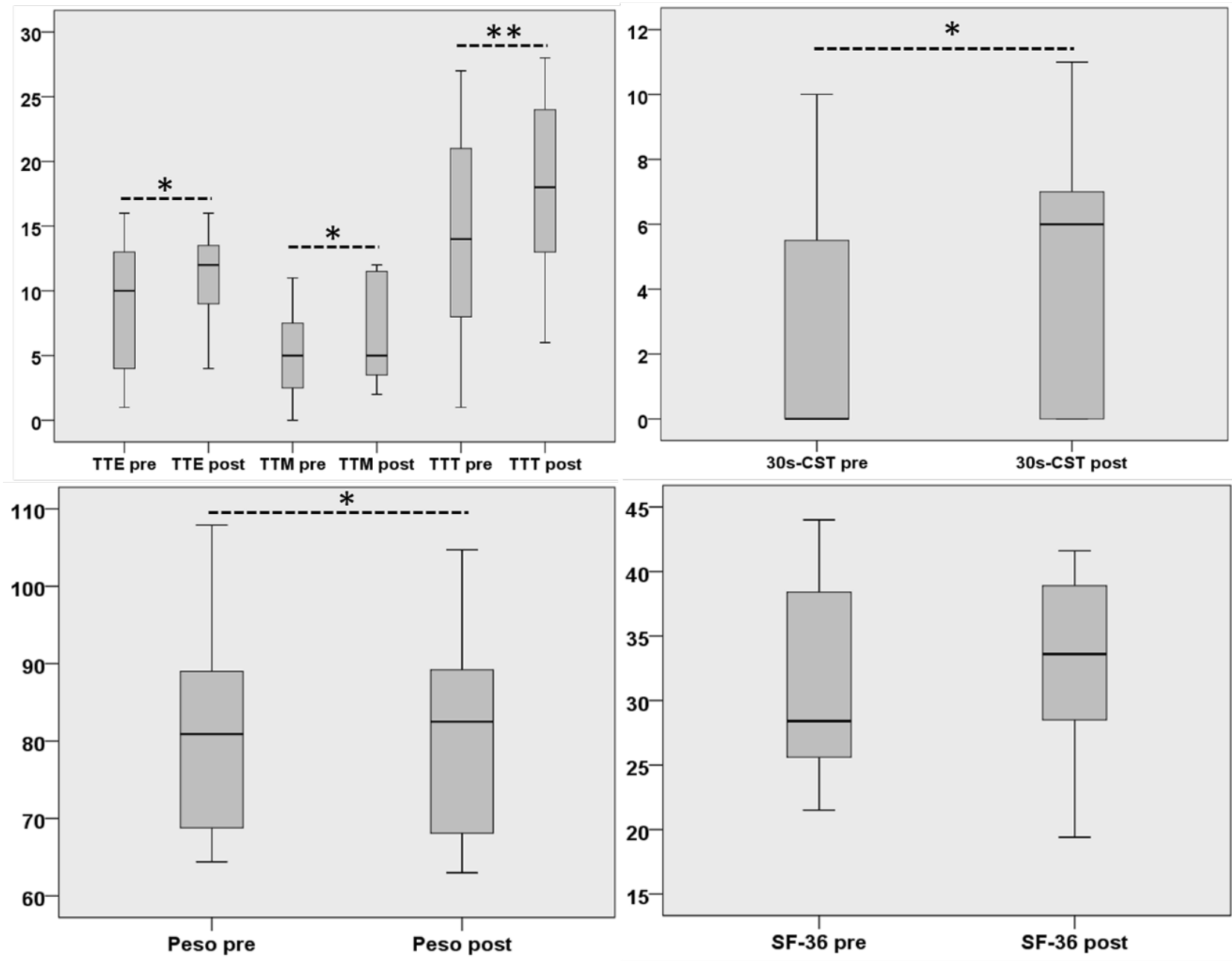

Figura 1. Diagrama de cajas. Valores pre-post tras cuatro semanas de entrenamiento neuromotor (programa EFAM$U V^{\odot}$ adaptado al ámbito hospitalario) sobre las variables. Los datos se presentan con la mediana (línea central), cuartiles primero y tercero (bordes externos de la caja), y valores extremos (mínimos y máximos) representados con las barras de error.

Superior izquierda: Tinetti Test (puntos en test de equilibrio: TTE; marcha: TTM; y Total: TTT).

Superior derecha: 30s-CST: 30-seconds Chair Stand Test (n ${ }^{\circ}$ de veces que se sienta y se levanta en 30 s). Inferior izquierda: Peso (Kg). ( $\mathrm{n}=9$; dos pacientes no pudieron pesarse sin ayuda). Inferior derecha: SF-36: Cuestionario de Salud SF-36 v2 (puntuación total).

$* \mathrm{p}>0.05 ; * * \mathrm{p}>0.01$ 
Blasco-Lafarga, C., Sanchis-Sanchis, R., Sanchis-Soler, G., San Inocencio-Cuenca, D., Llorens, P.

El hecho de reducir significativamente el peso, si bien no se ha conseguido con el resto de variables de la composición corporal, alienta la idea de conseguir mayores beneficios en intervenciones a más largo plazo, dado el carácter limitante del sobrepeso sobre la funcionalidad del adulto mayor, aunque no es objetivo del estudio.

Por otro lado, el registro de mejoras significativas en la ejecución del $T U G$ apunta a que la categorización propuesta puede ser adecuada para evaluar la agilidad en poblaciones con niveles físico-funcionales muy bajos. De nuevo, surge la necesidad de comprobar dicha propuesta y corroborar los beneficios del programa mediante un ensayo clínico con mayor tamaño muestral y con grupo control. Dada la importancia de este test como predictor de dependencia en las actividades de la vida diaria en adultos mayores (Donoghue, Savva, Cronin, Kenny, y Horgan, 2014), su categorización puede ser de gran utilidad en estudios futuros. Esta perspectiva algo más cualitativa puede ser especialmente importante en este tipo de población, donde un pequeño avance en su autonomía puede implicar una mejora en su autopercepción de competencia.

En este sentido, existe una relación probada y positiva entre la participación en los programas de actividad física y el aumento de la autoeficacia, con consecuencias sobre la mayor calidad de vida en los adultos mayores (Remor y Martins de Lima, 2009; Teixeira, Nunes, Ribeiro, Arbinaga, y VasconcelosRaposo, 2016). Aunque en nuestro estudio 4 semanas han sido insuficientes para mejorar la forma en que los participantes en el programa perciben su mejora en la calidad de vida relacionada con la salud, se observa un desplazamiento positivo de la mediana y una reducción del rango intercuartil de igual signo en el SF-36 (Figura 1). Por un lado, la situación cercana a la dependencia en estos pacientes (Tabla 1) hace difícil que las mejoras repercutan tan a corto plazo, por lo que debieran haberse considerado de forma complementaria instrumentos como la Escala de Autoestima o la Escala de Satisfacción con la vida, tal y como proponen Remor y Martins de Lima (2009). Por otro lado, la brevedad del programa, su orientación neuromuscular y la imposibilidad de trabajar con intensidades de moderadas a altas dificultan beneficios como los cambios hormonales que relacionan bienestar y ejercicio físico, fundamentalmente el ejercicio aeróbico, en programas de mayor duración (Carazo Vargas, Araya Vargas, y Salazar Rojas, 2006). Sin embargo, estos mismos autores señalan que los programas que incluyen fuerza influyen de forma similar sobre una capacidad mediadora tan importante como la capacidad cognitiva (tamaño del efecto de 0,45 en la orientación bioenergética frente a 0,44 en los programas de fuerza, o 0,31 cuando se combinan). De la misma forma se conocen los beneficios de la actividad física sobre la contención de la depresión asociada al envejecimiento y a la pérdida de capacidades y/o la soledad, que es otro factor clave en el mantenimiento de la calidad de vida en el adulto mayor (Barriopedro, Eraña, y Mallol, 2001; Stein, Molinero, Salguero, Corrêa, y Márquez, 2014; Teixeira et al., 2016), lo que de nuevo abre la puerta a los posibles beneficios sobre la calidad de vida de programas de este tipo en la UHD, si pudieran desarrollarse a más largo plazo, o varias veces al año.

Finalmente, la presencia del profesional en el domicilio ha supuesto un mayor grado de cumplimiento frente a las sesiones no supervisadas (estas últimas estaban controladas por un familiar o cuidador), superando el $80 \%$ exigido, lo que puede ser de gran importancia para estas poblaciones, con problemas de adherencia (Deka, Pozehl, Williams, y Yates, 2017).

Como ya se ha mencionado, al tratarse de un estudio piloto, la duración de la intervención fue corta (4 semanas de entrenamiento y 2 de evaluación) y el tamaño de la muestra pequeño, con asignación probabilística. El hecho de no disponer de grupo control con el que comparar los cambios igualmente reduce la extensión de estas conclusiones. Sin embargo, a pesar de sus limitaciones, estos resultados avalan el interés de los programas neuromotores en las UHD, y permiten pensar en la posibilidad de integrar su prescripción junto al resto del tratamiento pautado.

\section{APLICACIONES PRÁCTICAS}

De acuerdo con los resultados obtenidos en el presente trabajo, parece que estos programas desarrollados por equipos multidisciplinares son viables y pueden contribuir a mejorar la dimensión preventiva del sistema sanitario, aunque futuras intervenciones de mayor duración, con mayores tamaños muestrales, 


\section{Entrenamiento Neuromotor en pacientes ancianos pluripatológicos}

evaluadores ciegos y grupo control deberían dar soporte a estas afirmaciones. Igualmente se deberían establecer programas con diferente orientación, socializadores $\mathrm{u}$ otros complementarios que permitieran comparar los efectos respecto a esta propuesta neuromotora de ejercicio específica para el adulto mayor.

Dado el rápido efecto del desentrenamiento encontrado en poblaciones similares cuando cesa el ejercicio supervisado (Sanchis-Soler, 2017), se justifica la necesidad de intervenciones continuas en ancianos pluripatológicos con mermas importantes en la funcionalidad. Estas intervenciones podrían enriquecerse potenciando otras orientaciones como la cognitiva, la socioafectiva o la educativa, acentuando así su carácter formativo o de recordatorio sobre la importancia de mantener la actividad física en las poblaciones de ancianos pluripatológicos bajo supervisión de la UHD, incluso en momentos de exacerbación de la enfermedad. Más allá de mejorarla, contener la pérdida de funcionalidad del mayor y dotarlo de formación y herramientas para que lo pueda intentar por sí mismo también puede resultar fundamental en estas etapas de la vida.

\section{Agradecimientos}

Al personal sanitario de la UHD del Hospital General de Alicante, por su inestimable ayuda. A todos los pacientes que formaron parte de este proyecto. Y al Dr. Javier Martín-Sánchez por la revisión de este trabajo.

\section{REFERENCIAS}

1. Abizanda Soler, P. (2010). Actualización en fragilidad. Revista Española de Geriatría y Gerontología, 45(2), 106-110. doi: 10.1016/j.regg.2009.10.010

2. Alonso, J., Prieto, L., y Antó, J. (1995). La versión española del SF-36 Health Survey (Cuestionario de Salud SF-36): un instrumento para la medida de los resultados clínicos. Med Clin (Barc), 104(20), 771-776.
3. Asmidawati, A., Hamid, T. A., Hussain, R. M., y Hill, K. D. (2014). Home based exercise to improve turning and mobility performance among community dwelling older adults: protocol for a randomized controlled trial. BMC Geriatrics, 14(1), 100. doi: 10.1186/1471-2318-14-100

4. Barriopedro, M. I., Eraña, I., y Mallol, L. (2001). Relación de la actividad física con la depresión y satisfacción con la vida en la tercera edad. Revista de psicología del deporte, 10(2), 239-246.

5. Blasco Lafarga, C., Cordellat Marzal, A., Martínez Navarro, I., Monteagudo Chiner, P., Roldan Aliaga, A., Sanchis Soler, G., y Sanchis Sanchis, R. (2016). Autores/as de los derechos de Propiedad Intelectual del "Método de entrenamiento funcional cognitivo neuromotor EFAM-UV", bajo la titularidad de la Universitat de València, con $\mathrm{N}^{\mathrm{o}}$ Registro: 156069, y Fecha de Registro: 24/10/2016.

6. Blasco-Lafarga, C., Sanchis-Sanchis, R., SanchisSoler, G., y Llorens-Soriano, P. (2014). Physical and functional improvements after 4 weeks of a functional training program for home hospitalization unit patients. Paper presented at the Physiology 2014 Congress, London, UK.

7. Blasco-Lafarga, C., Sanchis-Soler, G., SanchisSanchis, R., y Valencia-Peris, A. (2015). Problemática y retos de un programa de entrenamiento funcional domiciliario para ancianos pluripatológicos pacientes de UHD. En Área de Cultura y Deportes de la Diputación de Málaga (Ed.), Longevidad y Salud. Innovación en la Actividad Física. (pp. 644-654). Málaga, Spain.

8. Carazo Vargas, P., Araya Vargas, G., y Salazar Rojas, W. (2006). Metaanálisis sobre el efecto del ejercicio en el funcionamiento cognitivo en adultos mayores. Revista iberoamericana de psicología del ejercicio y el deporte, 1(2), 89-104.

9. Casas-Herrero, Á., Cadore, E. L., MartínezVelilla, N., y Izquierdo, M. (2015). El ejercicio físico en el anciano frágil: una actualización. Revista Española de Geriatría y Gerontología, 50(2), 74-81. doi: 10.1016/j.regg.2014.07.003 
Blasco-Lafarga, C., Sanchis-Sanchis, R., Sanchis-Soler, G., San Inocencio-Cuenca, D., Llorens, P.

10. Casas Herrero, A., y Izquierdo, M. (2012). Ejercicio físico como intervención eficaz en el anciano frágil. Anales del Sistema Sanitario de Navarra, 35(1), 69-85. doi: 10.4321/S113766272012000100007

11. Clegg, A., y Young, J. (2011). The Frailty Syndrome. Clinical Medicine (London, England), 11(1), 72-75. doi: 10.7861/clinmedicine.11-1-72

12. Clegg, A., Young, J., Iliffe, S., Rikkert, M. O., y Rockwood, K. (2013). Frailty in elderly people. The Lancet, 381(9868), 752-762. doi: 10.1016/S0140-6736(12)62167-9

13. Da Silva-Grigoletto, M., Viana-Montaner, B., Heredia, J., Mata, F., Peña, G., Brito, C., . . . García-Manso, J. (2013). Validación de la escala de valoración subjetiva del esfuerzo OMNI-GSE para el control de la intensidad global en sesiones de objetivos múltiples en personas mayores. Kronos, 12(1), 32-40.

14. Deka, P., Pozehl, B., Williams, M. A., y Yates, B. (2017). Adherence to recommended exercise guidelines in patients with heart failure. Heart Failure Reviews, 22(1), 41-53. doi: 10.1007/s10741-016-9584-1

15. Di Benedetto, S., Müller, L., Wenger, E., Düzel, S., y Pawelec, G. (2017). Contribution of neuroinflammation and immunity to brain aging and the mitigating effects of physical and cognitive interventions. Neuroscience \& Biobehavioral Reviews, 75, 114-128. doi: 10.1016/j.neubiorev.2017.01.044

16. Donoghue, O. A., Savva, G. M., Cronin, H., Kenny, R. A., y Horgan, N. F. (2014). Using Timed Up and Go and Usual Gait Speed to Predict Incident Disability in Daily Activities Among Community-Dwelling Adults Aged 65 and Older. Archives of Physical Medicine and Rehabilitation, 95(10), 1954-1961. doi: 10.1016/j.apmr.2014.06.008

17. Epelde, F., Iglesias-Lepine, M. L., y Anarte, L. (2012). En plena crisis económica: coste y efectividad de las unidades de estancia corta hospitalarias. Anales del Sistema Sanitario de
Navarra, 35, 469-475. doi: 10.4321/S113766272012000300013

18. Garrett, S., Elley, C. R., Rose, S. B., O'Dea, D., Lawton, B. A., y Dowell, A. C. (2011). Are physical activity interventions in primary care and the community cost-effective? A systematic review of the evidence. British Journal of General Practice, 61(584), e125-e133. doi: 10.3399/bjgp11X561249

19. Guirao Martínez, R., Sempere Selva, M. T., López Aguilera, I., Sendra Pina, M. P., y Sánchez Payá, J. (2008). Unidad Médica de Corta Estancia, una alternativa a la hospitalización convencional. Revista Clínica Española, 208(5), 216-221. doi: $10.1157 / 13119913$

20. Hafström, A., Malmström, E.-M., Terdèn, J., Fransson, P.-A., y Magnusson, M. (2016). Improved Balance Confidence and Stability for Elderly After 6 Weeks of a Multimodal SelfAdministered Balance-Enhancing Exercise Program: A Randomized Single Arm Crossover Study. Gerontology and Geriatric Medicine, 2. doi: $10.1177 / 2333721416644149$

21. Izquierdo, M., Rodriguez-Mañas, L., CasasHerrero, A., Martinez-Velilla, N., Cadore, E. L., y Sinclair, A. J. (2016). Is It Ethical Not to Prescribe Physical Activity for the Elderly Frail? Journal of the American Medical Directors Association, 17(9), 779-781. doi: 10.1016/j.jamda.2016.06.015

22. Liubicich, M. E., Magistro, D., Candela, F., Rabaglietti, E., y Ciairano, S. (2012). Physical activity and mobility function in elderly people living in residential care facilities. "Act on aging": A pilot study. Advances in Physical Education, 2(2), 54-60. doi: 10.4236/ape.2012.22010

23. Mas, M. À., y Santaeugènia, S. (2015). Hospitalización domiciliaria en el paciente anciano: revisión de la evidencia y oportunidades de la geriatría. Revista Española de Geriatría y Gerontología, 50(1), 26-34. doi: 10.1016/j.regg.2014.04.003

24. Matsuda, P. N., Shumway-Cook, A., y Ciol, M. A. (2010). The Effects of a Home- Based Exercise Program on Physical Function in Frail Older 


\section{Entrenamiento Neuromotor en pacientes ancianos pluripatológicos}

Adults. Journal of Geriatric Physical Therapy, 33(2), 78-84. doi: 10.1097/JPT.0b013e3181deff9e

25. Ollero-Baturone, M., Sanz-Amores, R., y PadillaMarín, C. (2016). Plan andaluz de atención integrada a pacientes con enfermedades crónicas. Sevilla: Junta de Andalucía [consultado 11 de noviembre de 2018]. Disponible en: http://www.juntadeandalucia.es/salud/export/sites /csalud/galerias/documentos/p_2_p_2_planes_int egrales/PIEC.pdf

26. Pedersen, B. K., y Saltin, B. (2015). Exercise as medicine - evidence for prescribing exercise as therapy in 26 different chronic diseases. Scandinavian Journal of Medicine \& Science in Sports, 25, 1-72. doi: 10.1111/sms.12581

27. Remor, E., y Martins de Lima, S. (2009). Efecto diferencial del componente lúdico dentro de un programa específico de ejercicio físico para personas mayores sobre la autoestima, autoeficacia y satisfacción con la vida. Revista Iberoamericana de Psicología del Ejercicio y el Deporte, 4(2), 233-252.

28. Rikli, R. E., y Jones, C. J. (1999). Development and Validation of a Functional Fitness Test for Community-Residing Older Adults. Journal of Aging and Physical Activity, 7(2), 129-161. doi: 10.1123/japa.7.2.129

29. Rikli, R. E., y Jones, C. J. (2013). Development and validation of criterion-referenced clinically relevant fitness standards for maintaining physical independence in later years. The Gerontologist, 53(2), 255-267. doi: 10.1093/geront/gns071

30. Roldán-Jiménez, C., Bennett, P., y Cuesta-Vargas, A. I. (2015). Muscular Activity and Fatigue in Lower-Limb and Trunk Muscles during Different Sit-To-Stand Tests. PLOS ONE, 10(10), e0141675. doi: 10.1371/journal.pone.0141675

31. Sanchis-Soler, G. (2017). Incidencia del programa de entrenamiento cognitivo EFAMUV৫ en pacientes de la unidad de hospitalización a domicilio. Tesis doctoral, Universidad de Valencia, Valencia, España. Recuperado de http://roderic.uv.es/handle/10550/59128
32. Saüch, G., y Castañer, M. (2014). Observación de patrones motrices generados por los programas de actividad física para la tercera edad y la percepción de sus usuarios. Revista de Psicología del Deporte, 23(1), 181-190.

33. Sisamón, M. (2012). Prevención del déficit funcional en pacientes ancianos hospitalizados por enfermedad aguda: estudio preliminar de un programa de fuerza. Tesis doctoral, Universidad de Valencia, Valencia, España. Recuperado de http://roderic.uv.es/handle/10550/24617

34. Stein, A. C., Molinero, O., Salguero, A., Corrêa, M. C. R., y Márquez, S. (2014). Actividad física y salud percibida en pacientes con enfermedad coronaria. Cuadernos de Psicología del Deporte, 14(1), 109-116.

35. Teixeira, C. M., Nunes, F., Ribeiro, F., Arbinaga, F., y Vasconcelos-Raposo, J. (2016). Atividade física, autoestima e depressão em idosos. Cuadernos de Psicología del deporte, 16(3), 5566.

36. Tinetti, M. E., Franklin Williams, T., y Mayewski, R. (1986). Fall risk index for elderly patients based on number of chronic disabilities. The American Journal of Medicine, 80(3), 429-434. doi: 10.1016/0002-9343(86)90717-5

37. Vogel, T., Brechat, P. H., Leprêtre, P. M., Kaltenbach, G., Berthel, M., y Lonsdorfer, J. (2009). Health benefits of physical activity in older patients: a review. International Journal of Clinical Practice, 63(2), 303-320. doi: 10.1111/j.1742-1241.2008.01957.x

38. Woods, J. A., Vieira, V. J., y Keylock, K. T. (2009). Exercise, Inflammation, and Innate Immunity. Immunology and Allergy Clinics of North America, 29(2), 381-393. doi: 10.1016/j.iac.2009.02.011

39. Yates, L. B., Djoussé, L., Kurth, T., Buring, J. E., y Gaziano, J. (2008). Exceptional longevity in men: Modifiable factors associated with survival and function to age 90 years. Archives of Internal Medicine, 168(3), 284-290. doi: 10.1001/archinternmed.2007.77 